\title{
Structural, Atomic and Electrostatic Force Microscopy Analyses on YBCO/PBCO/LCMO Superlattices
}

\author{
Marcel Miyamura Bonilha ${ }^{a}$, Dayse Iara dos Santos ${ }^{a, b} *$, Nelcy Della Santina Mohallem ${ }^{c}$,
}

Luciana Moreira Searac ${ }^{c}$,Anne Hitomi Yonamine ${ }^{b, d}$

\author{
${ }^{a}$ Graduate Program in Science and Technology of Materials, São Paulo State University - UNESP, \\ CEP 17033-360, Bauru, SP, Brazil \\ ${ }^{b}$ Department of Physics, Faculty of Science, São Paulo State University - UNESP, CEP 17033-360, \\ Bauru, SP, Brazil \\ c Department of Chemistry, Federal University of Minas Gerais - UFMG, CEP 31270-901, \\ Belo Horizonte, $M G$, Brazil \\ ${ }^{d}$ Institute of Exact Sciences and Technology, Paulista University - UNIP, CEP 17048-290, Bauru, SP, Brazil
}

Received: September 25, 2016; Revised: January 25, 2017; Accepted: March 10, 2017

\begin{abstract}
In order to study the influence of the insulator layer thickness in heterojunctions, $\left(\mathrm{YBa}_{2} \mathrm{Cu}_{3} \mathrm{O}_{7-\delta[20 \mathrm{~mm}]} /\right.$ $\left.\mathrm{PrBa}_{2} \mathrm{Cu}_{3} \mathrm{O}_{\mathrm{y}} / \mathrm{La}_{1 / 3} \mathrm{Ca}_{2 / 3} \mathrm{MnO}_{3[20 \mathrm{~nm}]}\right)_{x 20}$ superlattices were prepared by pulsed laser deposition using three $\mathrm{PrBa}_{2} \mathrm{Cu}_{3} \mathrm{O}_{\mathrm{y}}$ layer thicknesses and two different sequences of deposition. Sample characterization showed primitive orthorhombic crystalline arrangement for YBCO and LCMO, however, a slightly disordered crystalline structure was observed for the sample having thicker PBCO layer. Microscopy analyses indicated influence of both parameters (PBCO thickness and sequence of deposition) on the texture of the upper layer. Electrostatic Force Microscopy analyses showed evident contrast on the phase images, what suggests that samples are conductive. Distinct surface aspect and highest contrast (highest shift in the electric mode phase image) were observed for the surface of the sample in which the sequence of deposition was inverted, with the YBCO ceramic as upper layer of the superlattice.
\end{abstract}

Keywords: Superconductors, Superlattices, Structural Analysis, AFM and EFM

\section{Introduction}

Among the superconducting devices, the $\mathrm{YBa}_{2} \mathrm{Cu}_{3} \mathrm{O}_{7-\delta}$ superlattices have been one of the most focused objects of research $^{1-5}$. In general, the aiming of the studies are the creation and design of new architectures of layers that can offer unusual electrical and magnetic characteristics. In the superlattices presented on this paper, the properties of the junctions are resultant of the proximity between the superconducting ceramic and the ferromagnetic manganite. The interaction between these distinct layers, under special conditions of temperature, magnetic field and the application of electric current, has proved to produce unexpected magnetization and resistivity responses that may be mediated by a layer of insulator material. There are experiments that prove that there is a range of proximity in which the layers may affect each other more or less intensively depending on the thickness and the nature of the insulator layer introduced between them. Consequently, it should be possible to tailor the properties of the superlattice using a defined separator with a precise thickness. For example, in the case of a ferromagnet-superconductor junction, it can be assumed that there is a diffusion length $\xi$ within which the intensified field on the ferromagnet side advances toward the superconducting layer destroying the coupling between the Cooper Pairs of opposite spins ${ }^{6}$. Since

\footnotetext{
* e-mail: dayse@fc.unesp.br
}

the interface between the materials constitutes the most part of the superlattice, the extent of interaction between these layers can determine the behavior of the entire composite ${ }^{7}$. In the latter years, the phenomenon of colossal magnetoresistance ${ }^{8,9}$ was revealed in some of those experiments with superlattice made of certain junctions and tested at specific conditions, which is quite interesting for their technological applications.

Inside a major project ${ }^{8}$, a set of superlattices was made of $\mathrm{YBa}_{2} \mathrm{Cu}_{3} \mathrm{O}_{7-\delta}(\mathrm{YBCO})$ superconducting ceramic interacting with $\mathrm{La}_{2 / 3} \mathrm{Ca}_{1 / 3} \mathrm{MnO}_{3}$ (LCMO) manganite ferromagnetic ultrafine layers of the same thicknesses. For mediating and investigating the intensity and the type of their interactions, it was deposited an insulation layer, like $\mathrm{PrBa}_{2} \mathrm{Cu}_{3} \mathrm{O}_{\mathrm{y}}(\mathrm{PBCO})$, between the superconductor and the manganite. As one can see, the selected insulator is also a cuprate, but it can be considered as a normal ceramic since its superconducting transition requires special processing, which was not provided. In this previous study ${ }^{8}$, it was observed that their novel electric/magnetic properties as well as structural/ textural characteristics and also electrical conduction were dependent on the insulator thickness. Therefore, a better structural characterization of the superlattices to find out if there was a correlation between the structural characteristics, the insulator layer thickness and their unusual electric/ magnetic properties is necessary. 
Finally, this paper reports analyses of X-ray diffraction, scanning electron and atomic force microscopies that were investigated for $\left(\mathrm{YBCO}_{20 \mathrm{~mm}} / \mathrm{PBCO} / \mathrm{LCMO}_{20 \mathrm{~nm}}\right)_{x 20}$ superlattices having PBCO layers with $0.5 \mathrm{~nm}, 8 \mathrm{~nm}$ and $20 \mathrm{~nm}$ thicknesses and one distinct sample which was prepared with the inversion of the deposition sequence of YBCO and LCMO layers.

\section{Experimental}

The superlattices were prepared by pulsed laser deposition (PLD) technique, using a KrF excimer laser with $\lambda=248 \mathrm{~nm}$, with excitation flow of $3-4 \mathrm{~J} / \mathrm{cm}^{2}$ and frequency of $5 \mathrm{~Hz}$. The deposition chamber was kept at $10^{-6}$ torr of pressure and $1033 \mathrm{~K}$ of temperature. A flow of $\mathrm{O}_{2}$ (300mtorr) was inserted during the layers deposition. First, a buffer layer of $\mathrm{SrTiO}_{3}(\mathrm{STO})$ was deposited on the $\mathrm{LaAlO}_{3}(001)$ substrate (LAO). Then, the deposition sequence was $\mathrm{YBCO} / \mathrm{PBCO} / \mathrm{LCMO}$, which was repeated 20 times. Before each one of this sequence, a PBCO layer was deposited. The thicknesses of YBCO and LCMO layers were always $20 \mathrm{~nm}$, while the PBCO thickness was chosen to be $0.5 \mathrm{~nm}, 8 \mathrm{~nm}$ and $20 \mathrm{~nm}$, for each sample. An extra sample, with $8 \mathrm{~nm}$ thickness of $\mathrm{PBCO}$, was prepared with the sequence switched to $\mathrm{LCMO} / \mathrm{PBCO} / \mathrm{YBCO}$ in order to observe any influence on its properties. As showed in Table 1 , the samples were denoted by a letter citing the compound in the upper layer of the superlattice ( $\mathrm{L}$ for LCMO and $\mathrm{Y}$ for YBCO) and the thickness of the PBCO film.

Table 1: Notation and description of the samples.

\begin{tabular}{lcc}
\hline Sample & $\begin{array}{c}\text { PBCO layer thickness } \\
(\mathrm{nm})\end{array}$ & $\begin{array}{c}\text { Estimated total thickness } \\
(\mathrm{nm})\end{array}$ \\
\hline L05 & 0.5 & 820 \\
L8 & 8 & 1120 \\
Y8 & 8 & 1120 \\
L20 & 20 & 1600 \\
\hline
\end{tabular}

Obs.: The letters $\mathrm{L}$ and $\mathrm{Y}$ denote that the upper lattice layer is LCMO or YBCO, respectively.

The X-ray diffraction patterns of the samples were obtained by powder method ( $2 \theta / \theta$ configuration) using Rigaku

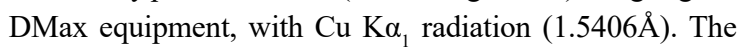
atomic and electrostatic force microscopies (AFM and EFM, respectively) were performed in an Asylum Research MFP 3D using intermittent-contact method. The EFM images were obtained by applying an electric potential of $3.0 \mathrm{~V}$ between the tip and the sample base. To each EFM image, a simultaneous topographic image was also generated. The scanning electron microscopy (SEM) was made in a Zeiss EVO-015 microscope.

\section{Results and Discussion}

By X-ray diffraction it was found that $\mathrm{YBCO}, \mathrm{PBCO}$ and LCMO are present in the structure and are arranged in the primitive orthorhombic lattice, as expected. For YBCO and $\mathrm{PBCO}$, it is known that their lattice parameters are very close and in these samples it was observed deviations of $\approx 1 \%$ for $a$ and $b$, and $<0.2 \%$ for $c$. In this way, the peaks that were indexed for YBCO were also valid for PBCO. According to crystallographic data, the $a$ and $b$ parameters of LCMO are quite different from those of the cuprates but are still compatible considering the diagonal of the $a-b$ plane of YBCO and PBCO. The experimental diffractograms showed in Figure 1 also revealed that the cited cuprates as well as the manganite were deposited with $(00 \ell)$ orientation in all studied superlattices. The $c$ parameter was calculated for these YBCO peaks and the dispersion was found to be lower than $0.9 \%$ for each plane and about $0.4 \%$ for each superlattice, when compared to the expected one for YBCO, $\mathrm{c}=11.680 \AA$.

In the case of L20 diffractogram, it was noticed significant broadening of the peaks due to the disordering introduced by the large growing of the layer.

The presence of peaks of the LAO substrate was also verified for all samples. But the L20 sample had the less intense peaks since the total thickness of the superlattice is estimated in $1.6 \mathrm{~mm}$, which is comparable to the limit of $\mathrm{X}$-ray penetration into the surface.

An estimation of the average crystallite size of YBCO is presented in Table 2 for each case, which was calculated using the full width half maximum (FWHM) of Y(007) peak in the Scherrer equation ${ }^{10}$. This peak was selected for this calculation for it is the most clear and visible YBCO one in all diffractograms. Although there was no clear correlation between the crystallite size and the PBCO thickness, it was visible the influence of the sequence of deposition, since it was found crystallite mean size about $9 \mathrm{~nm}$ for $\mathrm{L} 8$ and $15 \mathrm{~nm}$ for Y8. This fact indicates better YBCO crystal growth orientation when LCMO is deposited firstly on the STO buffer layer, which is also evidenced by the decrease of the $\mathrm{Y}(207)$ relative intensity peak observed for this superlattice.

The images of the upper surfaces that were captured using the atomic and the electrostatic force microscopy at room temperature are presented in left and right columns, respectively, in Figure 2. In the first case, the AFM measurement, the microscope evidenced the topography of the LCMO superlattice surface. This procedure is usually based in the Van der Waals force, having short range interaction between the microscope tip and the sample. No long distance Coulomb interaction was acting because no electric field was applied. In the left column it is possible to notice islands growth along with the PBCO thickness.

As seen, the grains grew from a few nanometers in size, for L05, to about $500 \mathrm{~nm}$ agglomerates for L20. The association of the average size of the islands with the hollow space between them corresponds to the roughness (RMS). The RMS was determined for each sample considering the exposed area in Figure 2, and they were estimated to be 


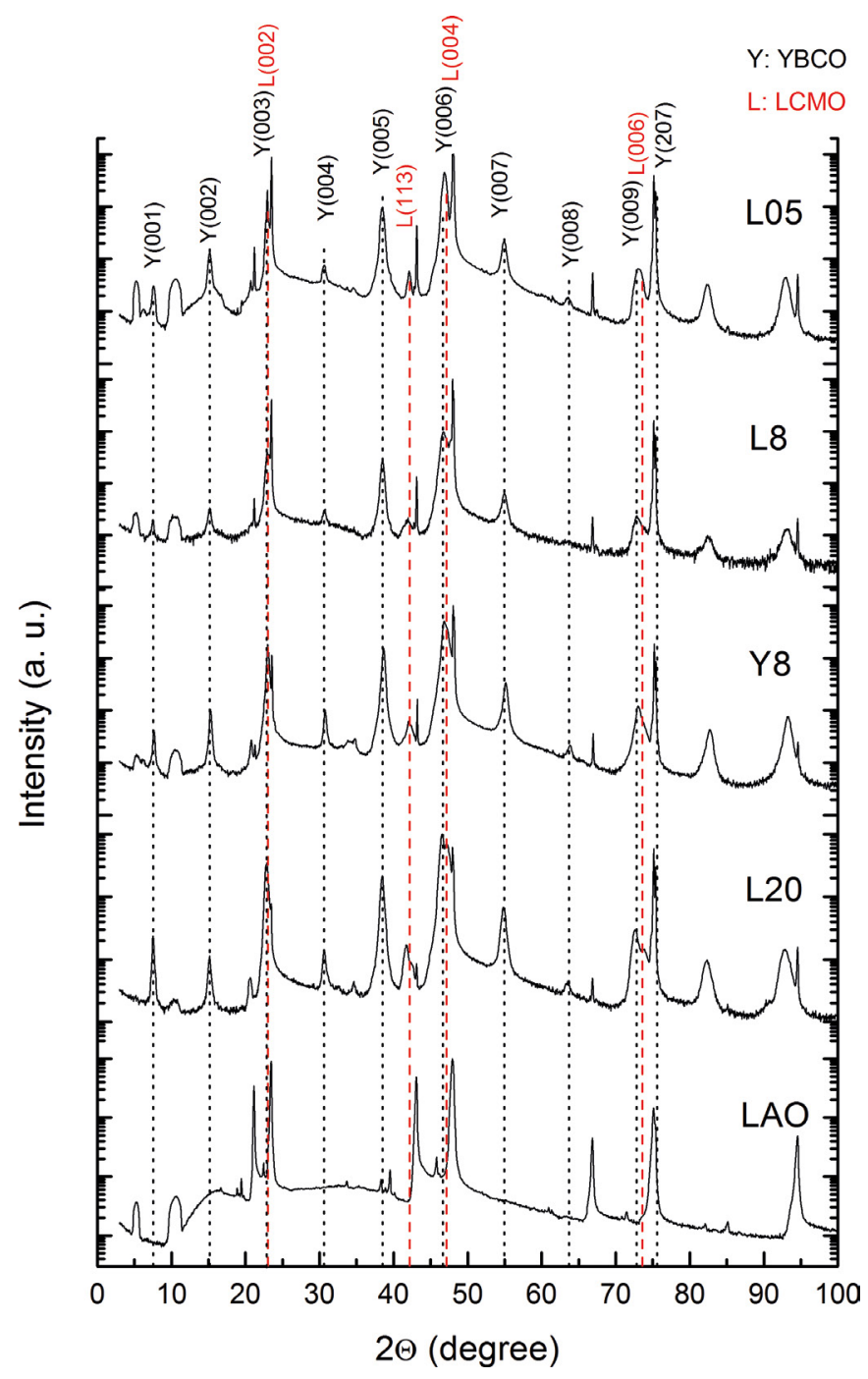

Figure 1. Diffractograms of the superlattices with $0.5 \mathrm{~nm}$ (L05), $8 \mathrm{~nm}$ (L8 and Y8) and 20nm (L20) PBCO layers, and the substrate $\mathrm{LaAlO}_{3}$ (001) (LAO).

Table 2: Full width half maximum (FWMH) of Y(007) peak, estimated average crystallite size of YBCO and roughness (RMS) obtained for each superlattice.

\begin{tabular}{lccc}
\hline Sample & FWMH (rad) & $\tau(\mathrm{nm})$ & RMS(nm) \\
\hline L05 & 0.015 & 10.3 & 25.6 \\
L8 & 0.018 & 8.8 & 10.1 \\
Y8 & 0.011 & 14.6 & 28.8 \\
L20 & 0.013 & 12.0 & 4.5 \\
\hline
\end{tabular}

Obs.: The estimation of the average crystallite size of YBCO was performed without considering the deformation introduced by the $\mathrm{X}$-ray diffraction equipment.

between $25.6 \mathrm{~nm}$ and $4.5 \mathrm{~nm}$ for the LCMO toped samples. The lowest roughness was found for L20, which showed largest grains in the AFM image and higher crystallite growth observed by X-ray diffraction.

The small white spots on top of the L20 are silver particles left from previous electrical measurements that could not be removed.
Pores and unleveled grains are quite noticed for sample L05, and irregular hollows between the islands are observed for the samples L8 and L20.

The large chunks on top of all samples are made of the same compounds launched by the plume and were due to small fluctuations of the conditions inside the deposition chamber ${ }^{11,12}$.

According to Masilamani et al. ${ }^{13}$, in their search for buffer layer materials for YBCO thin film devices having smooth top surfaces, the PBCO layer was the structurally and chemically best compatible material for YBCO among the tested materials. This layer, having approximated $30 \mathrm{~nm}$ thickness, has promoted significant improvement of the surface morphology of the film, although it was also responsible for a slight increase on the critical temperature transition width due to a small inhomogeneity in the YBCO thin film. The study, however, was not extended for different PBCO layer thicknesses as it is presented here in this paper for superlattices. 

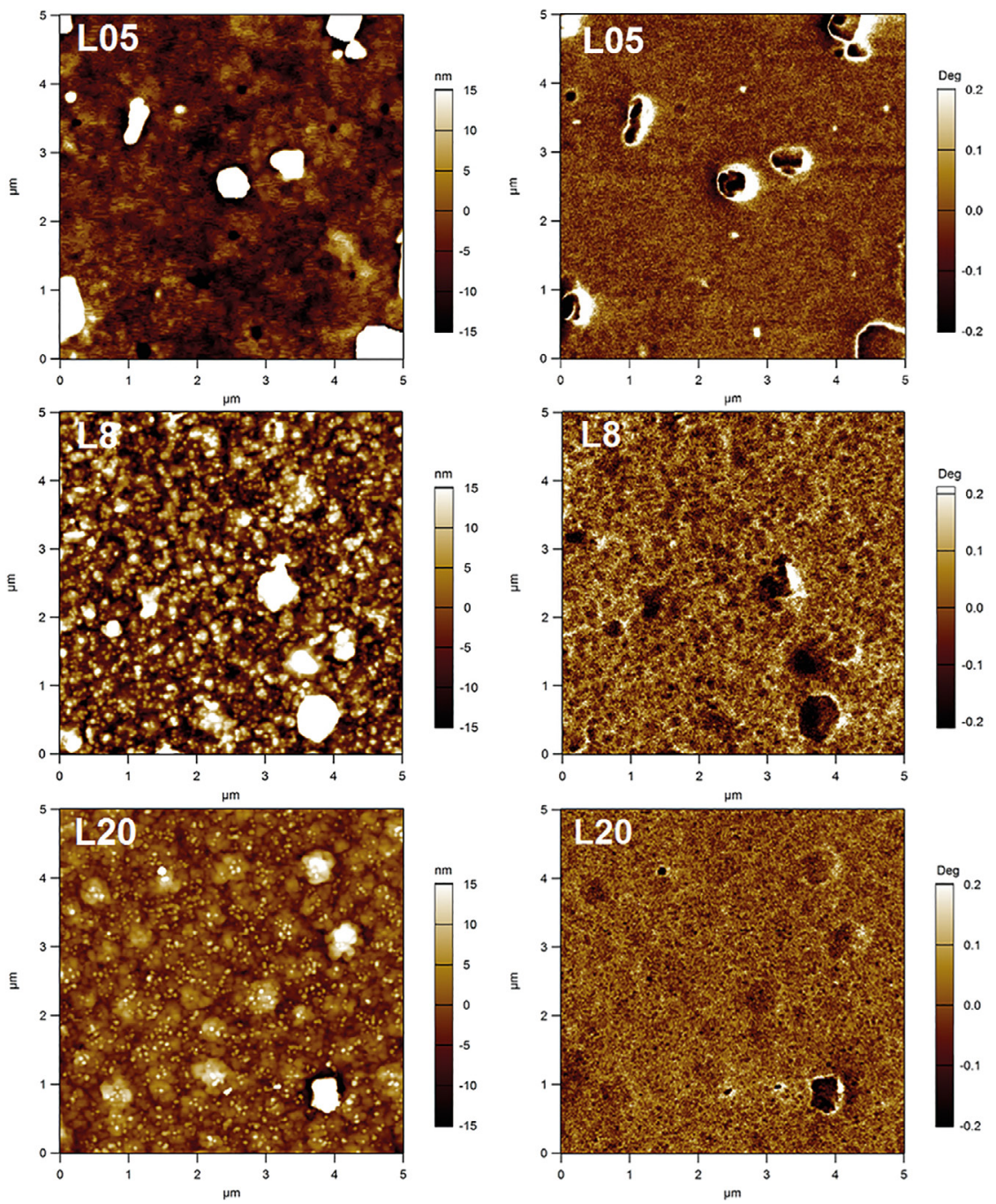

Figure 2. Images showing the topography obtained by AFM (left column) and the mapping of charge conduction, obtained by phase EFM (right column), for $5 \mu \mathrm{m} \times 5 \mu \mathrm{m}$ regions of the LCMO top layer.

By the other hand, for $\mathrm{YBCO} / \mathrm{PBCO} / \mathrm{LCMO}$ superlattices, the images obtained by microscopies revealed that the microstructure and texture of the upper layer, and probably the layers underneath, are dependent on the PBCO layer thickness. In such a way that, based on the RMS results, the thicker this layer, the less rough and with fewer defects is the upper surface. Nevertheless, the number of repetitions of the $\mathrm{YBCO} / \mathrm{PBCO} / \mathrm{LCMO}$ sequence was not changed and it also should play an important role on the surface and underneath layers of the superlattice.

The images obtained by EFM measurements, in the right column of Figure 2, were resulting from long distance Coulomb interactions between the tip and the base of the sample when an electric potential of $3.0 \mathrm{~V}$ was applied. This procedure turned possible to verify that the samples were conductive, and also allowed the mapping of the regions of the surfaces where the shift of phase was more intense.
Accordingly to that, the L05 and L8 EFM images evidenced more intensively the islands seen in the AFM images and enhanced the most conductive spots, those which are highly saturated (dark islands). Unlike these samples, the L20 showed a different perspective and had not presented the large islands as in the left side of Figure 2. Low contrast seems to be the main characteristic for its EFM image shown by low shift in EFM phase, which may be an indication of lower electrical conductivity. Considering electrical (Four electrodes method) and magnetization measurements performed in previous work $^{8,14}$ on this set of samples, L20 superlattice presented quite small influence of the ferromagnetic material, LCMO, on the resulting superconducting properties, justified by the thick layer high PBCO shielding. Consequently, this superlattice produces very low incidence of unusual physical effects like Colossal Magnetoresistance, already observed for a few samples under special conditions. 
In Figure 3 only the superlattices having 8nm layer of PBCO are presented. The AFM images on the left column shows that the texture of the two surfaces are very distinct, agreeing to the fact that they are two different materials, LCMO and YBCO. Comparing the size of the islands and hollows for each of these samples, one notices wider island size and hollow distributions for Y8 than for L8. In this way, a rougher texture, presenting a RMS about $28.8 \mathrm{~nm}$, is observed for the YBCO top, in spite of presenting significant larger average crystallite size when comparing to the LCMO top of L8. However, this last parameter is evaluated for the whole superlattice, while the RMS data is estimated just for its top layer.

Besides that, as seen by the saturated spots in the images on the bottom right column, the Y8 superlattice showed higher contrast in EFM phase image. And this may be an indication of change in electrical conductivity effectively related to the sequence of deposition. In principle, the electric potential applied between the EFM tip and the base of the sample is exposing the same material (in nature and in thickness) in Y8 as in L8, but the overall conductivity seems to be changed. Actually, previous electrical resistivity measurements under $0.8 \mathrm{~T}$ applied magnetic field ${ }^{14}$ for $\mathrm{Y} 8$, revealed also more intense response to field application and orientation, besides higher stability, than the samples with LCMO on the top. But that fact could be attributed to better electrical contact between the electrodes and the YBCO top surface compared to the LCMO-electrodes contacts.

The corresponding 3D images of these two samples, L8 and Y8, are presented in Figure 4, where it is possible to notice more clearly the thinner texture of the LCMO compared with the top YBCO. The difference between the top microstructures may be representative of the respective underneath layers as well.

The SEM micrographs in Figure 5 are showing all the top surfaces studied in this paper at a $6,000 \mathrm{x}$ magnification. With an overall look it is possible to identify pores on LCMO and outgrowth on the YBCO surfaces. The excess of defects observed for L05 is due to the flaws caused by the deposition of this very thin layer of $\mathrm{PBCO}$, which is in
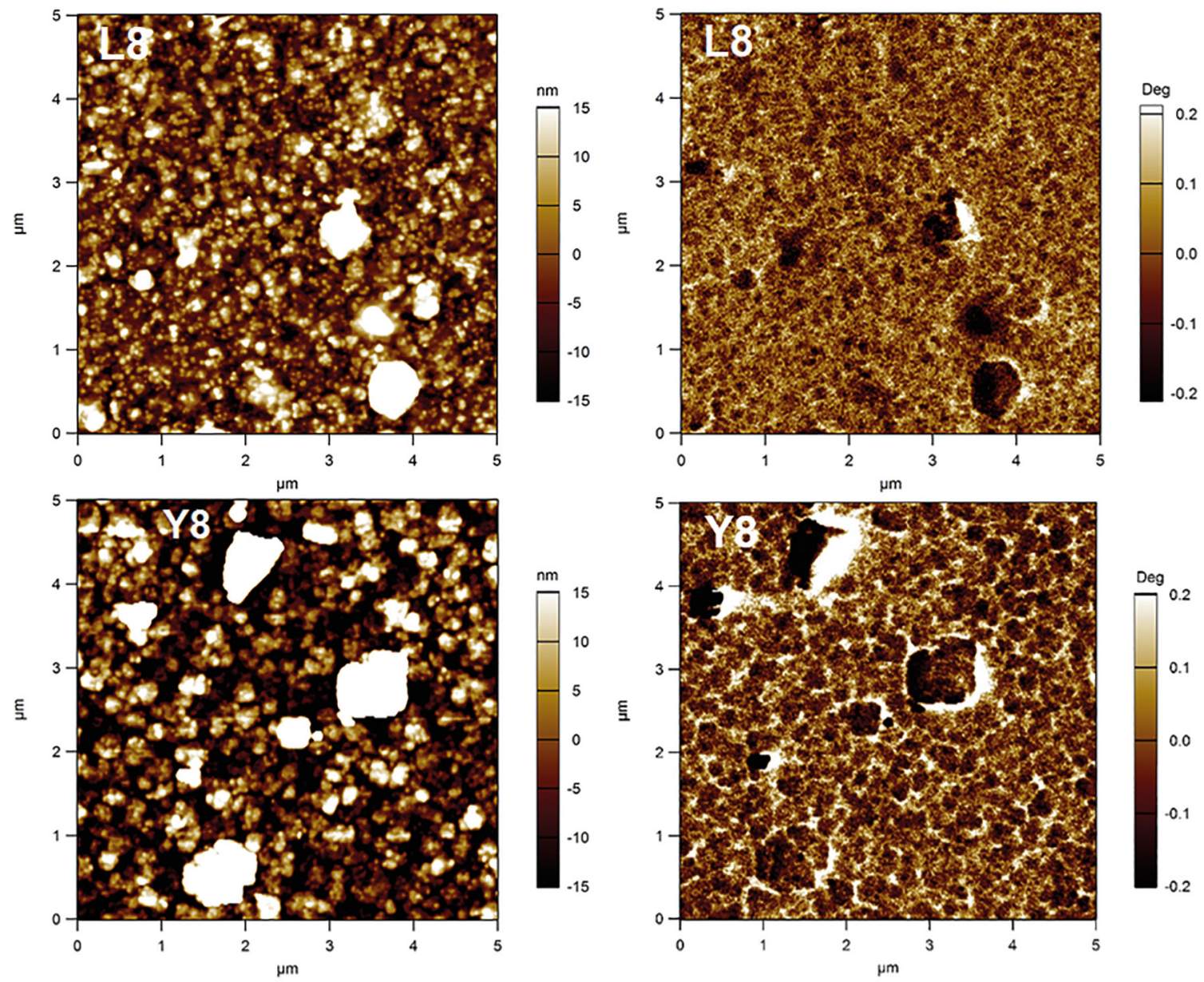

Figure 3. Topography obtained by AFM (left column) and the mapping of charge conduction, obtained by phase EFM (right column), for $5 \mu \mathrm{m} \times 5 \mu \mathrm{m}$ regions of the LCMO (L8) and YBCO (Y8) top layers for the superlattices having $8 \mathrm{~nm}$ layer of PBCO. 


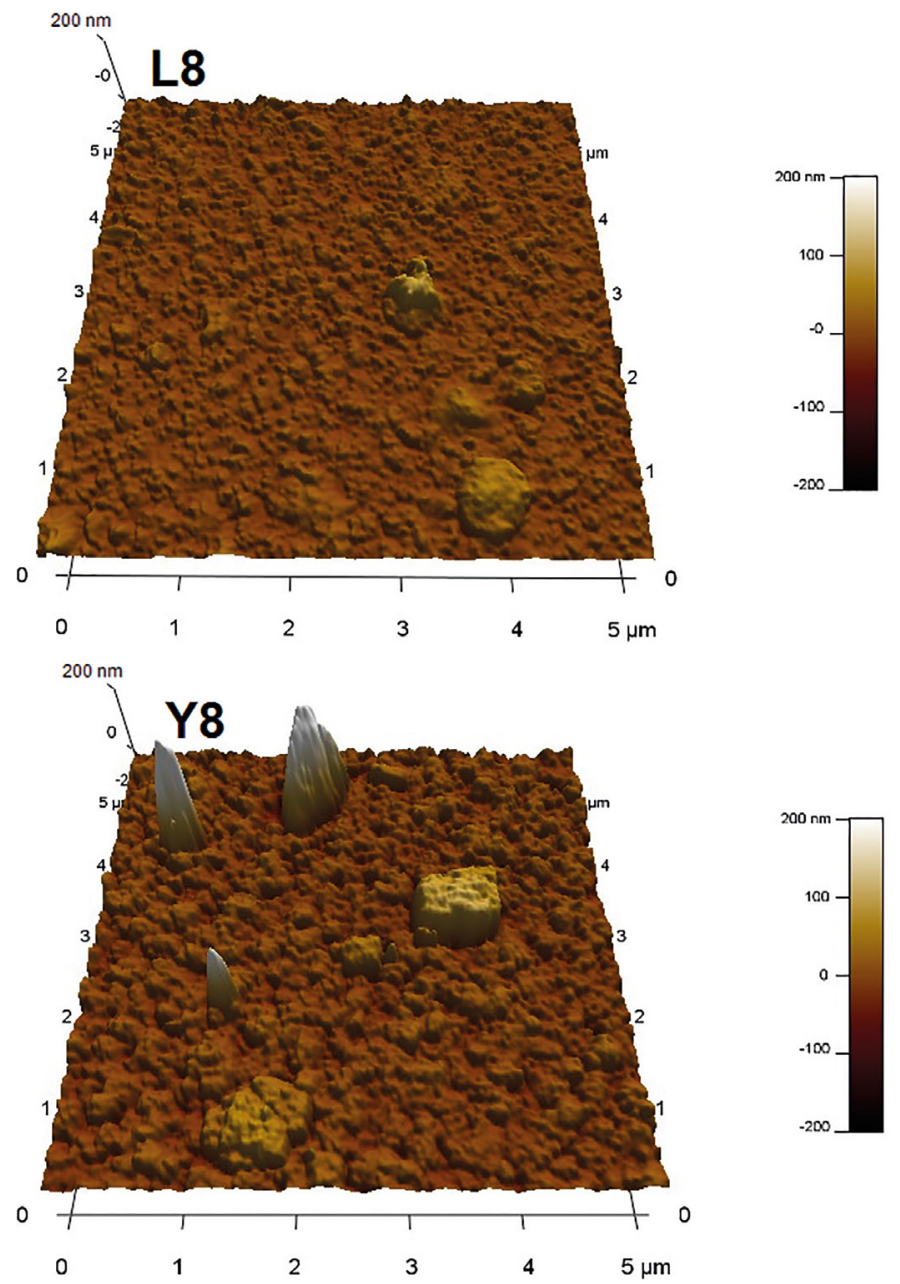

Figure 4. $3 \mathrm{D}$ topography obtained by AFM for $5 \mu \mathrm{m} \times 5 \mu \mathrm{m}$ regions of the LCMO (L8) and YBCO (Y8) top layers for the superlattices having 8nm layer of PBCO.

fact almost half monolayer of this cuprate. The SEM images under this magnification indicate that the surface of sample L20 presents the highest density of defects. By the other hand, energy dispersive spectroscopy performed for each sample evidenced the presence of all expected elements in the studied superlattices.

\section{Conclusions}

The XRD analysis reflects the structure of the entire superlattice, in depth and surface, and no great difference was found between the diffractograms of the samples. But, detailed observation reveals a slight broadening of the peaks indicating higher disorder for L20 and a variable average crystallite size for $\mathrm{Y}(007)$ peak calculated for each sample. A great deal of change was found between the average crystallite size for L8 and Y8, reflecting some improvement in the structure for the inverted sequence of deposition.

By the other hand, the images obtained by AFM microscopy in $5 \mathrm{~mm} \times 5 \mathrm{~mm}$ regions revealed that the microstructure and texture of the upper layer, and probably the layers underneath, are dependent on the PBCO layer thickness, becoming smoother for thicker ones. While an overall view made possible by scanning electronic microscopy over a $40 \mathrm{~mm}$ x $40 \mathrm{~mm}$ area showed increased surface degradation with thickness.

In turn, the electrostatic force microscopy indicated that all the superlattices are conductive but there is a tendency to be less conductive for thicker insulator layer. One important note is the higher saturation (dark spots) observed for Y8 compared to L8 in the EFM characterization (right column of in Figure 3), despite the fact that the two superlattices are made of the same materials and respective thicknesses. The difference between the LCMO and YBCO top layers, clearly seen by 3D AFM and phase EFM images, brings about the difference between the microstructures and conductive properties of the underneath respective layers and may bring some light about their PBCO mediated interaction as well, which turn these data quite important for the design of new superlattices with electronic devices purpose. 


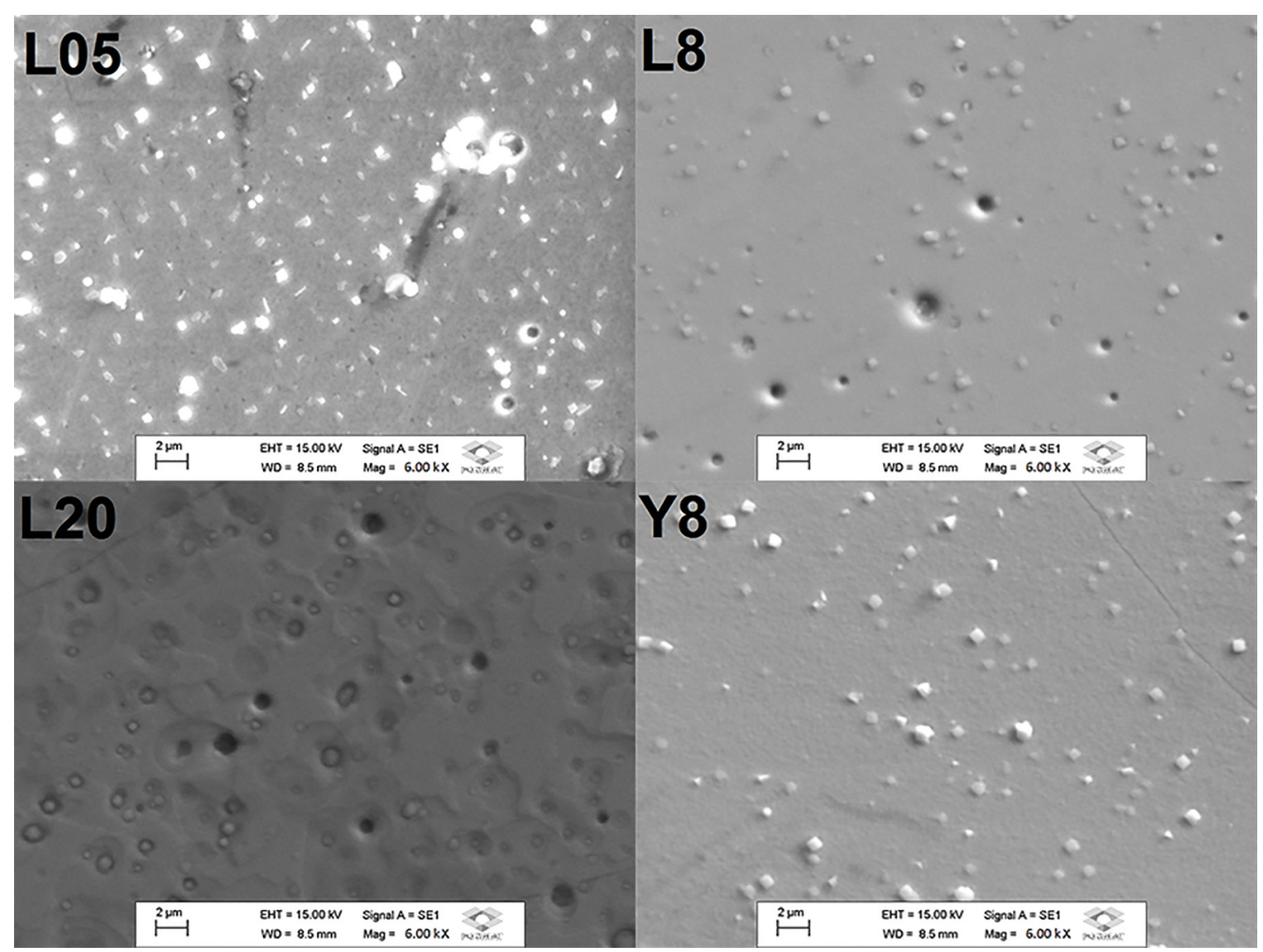

Figure 5. SEM micrographs of the surface of the superlattices $(6,000 \mathrm{x}$ magnification).

\section{Acknowledgments}

The authors wish to thank Dr. A. V. Pan and Dr. S. A. Fedoseev from Institute for Superconducting and Electronic Materials, University of Wollongong, Australia, for the facilities and confection of the superlattices. The authors thank the Multiuser Laboratory of the Department of Physics, UNESP Campus Bauru for the XRD and SEM measurements and the Center of Microscopy, UFMG, for the AFM/EFM analyses. The authors also thank Coordenação de Aperfeiçoamento de Pessoal de Nível Superior (Capes/Brazil) for the scholarship.

\section{References}

1. Hwang HY, Iwasa Y, Kawasaki M, Keimer B, Nagaosa N, Tokura Y. Emergent phenomena at oxide interfaces. Nature Materials. 2012;11(2):103-113.

2. Mannhart J, Schlom DG. Oxide interfaces-an opportunity for electronics. Science. 2010;327(5973):1607-1611.

3. Chakhalian J, Freeland JW, Habermeier HU, Cristiani G, Khaliullin, $\mathrm{G}$, van Veenendaal M, et al. Orbital reconstruction and covalent bonding at an oxide interface. Science. 2007;318(5853):1114-1117.
4. Zhang ZL, Kaiser U, Soltan S, Habermeier HU, Keimer B. Magnetic properties and atomic structure of $\mathrm{La}_{2 / 3} \mathrm{Ca}_{1 / 3} \mathrm{MnO}_{3}-\mathrm{YBa}_{2} \mathrm{Cu}_{3} \mathrm{O}_{7}$ heterointerfaces. Applied Physics Letters. 2009;95(24):242505.

5. Blagoev BS, Nurgaliev TK, Štrbik V, Mateev ES, Zaleski AJ. Magnetic and transport parameters of LSMO and YBCO/LSMO films deposited on sapphire substrates. eprint arXiv:1307.0302 [Internet]. 2013. Available from: <http://arxiv.org/abs/1307.0302>. Access in: 18/09/2016.

6. Soltan SEAG. Interaction of Superconductivity and Ferromagnetism in $Y B C O / L C M O$ Heterostructures. [PhD Thesis]. Stuttgart: Max Planck Institute; 2005.

7. Chien TY, Kourkoutis LF, Chakhalian J, Gray B, Kareev M, Guisinger $\mathrm{NP}$, et al. Visualizing short-range charge transfer at the interfaces between ferromagnetic and superconducting oxides. Nature Communications. 2013;4:2336.

8. Yonamine AH. Estudo da interação entre camadas de super-redes supercondutor/isolantelferromagneto. [ $\mathrm{PhD}$ Thesis]. Bauru: Universidade Estadual Paulista Júlio de Mesquita Filho; 2014. 162 f.

9. Fedoseev SA, Pan AV, Rubanov S, Golovchanskiy IA, Shcherbakova OV. Large, Controllable Spikes of Magnetoresistance in $\mathrm{La}_{2 / 3} \mathrm{Ca}_{1 / 3} \mathrm{MnO}_{3} /$ $\mathrm{SrTiO}_{3}$ Superlattices. ACS Nano. 2013;7(1):286-293.

10. Patterson AL. The Scherrer Formula for X-Ray Particle Size Determination. Physical Review. 1939;56(10):978-982. 
11. Aghabagheri S, Mohammadizadeh MR, Kameli P, Salamati H. Effect of Oxygen Pressure on the Surface Roughness and Intergranular Behavior of YBCO Thin Films. Journal of Superconductivity and Novel Magnetism. 2016;29(6):1483-1489.

12. Pan AV, Pysarenko S, Dou, SX. Drastic improvement of surface structure and current-carrying ability in $\mathrm{YBa}_{2} \mathrm{Cu}_{3} \mathrm{O}_{7}$ films by introducing multilayered structure. Applied Physics Letters. 2006;88(23):232506.
13. Masilamani N, Shcherbakova OV, Fedoseev SA, Pan AV, Dou SX. Effect of substrate and buffer layer materials on properties of thin $\mathrm{YBa}_{2} \mathrm{Cu}_{3} \mathrm{O}_{7-\mathrm{x}}$ films. IEEE Transactions on Applied Superconductivity. 2013;23(3):6601105.

14. Bonilha MM. Estudo das propriedades de super-redes YBCO/ isolante/LCMO. [MSc Dissertation]. Bauru: Universidade Estadual Paulista Júlio de Mesquita Filho; 2016. 